\title{
Review on Up-to-Date Status of Candidate Vaccines for COVID-19 Disease
}

This article was published in the following Dove Press journal:

Infection and Drug Resistance

Tafere Mulaw Belete

Department of Pharmacology, College of Medicine and Health Sciences, University of Gondar, Gondar, Ethiopia
Correspondence: Tafere Mulaw Belete $\mathrm{Tel}+251918045943$

Email mutafere@yahoo.com

\begin{abstract}
The global pandemic of COVID-19 caused by SARS-CoV-2 continues to spread and poses serious threats to public health and economic stability throughout the world. Thus, to protect the global population, developing safe and effective vaccines is mandatory to control the spread of SARS-CoV-2 pandemic. Since genomic sequences of SARS-CoV-2 and SARS-CoV-1 have similarity and use the same receptor (ACE2), it is important to learn from the development of SARS-CoV-1 vaccines for the development of SARS-CoV-2 vaccines. Normally vaccine development takes $10-15$ years but vaccine development against SARSCoV2 is going on at a very fast pace resulting in almost breakthrough methods of vaccine development by several research institutions. The whole process of vaccine development including clinical trials gets shortened and may be fast tracked to 15-18 months. Global collaborations and increased research efforts among the scientific community have led to more than 214 candidate vaccines globally. The current review highlights the different approaches and technologies used around the world for the design and development of the vaccines and also focuses on the recent status of the SARS-CoV-2 vaccine candidates under development by various institutions to combat the world threat of COVID-19 pandemic.
\end{abstract}

Keywords: COVID-19, SARS-CoV-2, clinical trials, vaccine, spike protein

\section{Background}

Viruses have the potential to cause irreparable loss to human beings. In 1960, coronavirus (Covs) was identified as the cause of the common cold. At the present time seven types of Covs species can infect human beings. The four Covs species (HCoV-NL63, HCoV-229E, CoV-HKU1, and HCoV-OC43) cause up to a third of community-acquired upper respiratory tract infections. The remaining three species are highly pathogenic; SARS-CoV-1 discovered in 2002 as the cause of severe acute respiratory syndrome (SARS), the Middle East respiratory syndrome Covs identified in 2012 as the cause of the Middle East respiratory syndrome (MERS), and SARS-CoV-2 as the cause of Covs disease 2019 (COVID-1). ${ }^{1}$ COVID-19 characterized by respiratory distress was first reported in December 2019, in the People's Republic of China. On March 11, 2020, the World Health Organization (WHO) declared COVID-19 as a pandemic disease. ${ }^{2}$ For example, the S protein in SARS-CoV-1 is highly conserved in SARS-CoV-2 and has great potential to induce $\mathrm{T}$ cell response. These similarities help to develop treatment interventions.

As of December 21, 2020, there were 75,479,805 cases and 1,686,267 confirmed deaths in the world. ${ }^{3}$ SARS-CoV-2 has stronger infectivity than that of SARS-CoV-1 and poses an immense threat to the world population. SARS-CoV-2 is highly contagious, spread by the inhalation of respiratory aerosols and direct human contact. ${ }^{4}$ The serious 
pandemic situation has highlighted the need for safe and effective therapeutic and preventive measures to reduce the transmission of the disease. Since effective antiviral agents are still lacking, vaccination is the best approach for controlling the current outbreak of SARS-CoV-2. ${ }^{5}$ Therefore, several countries have accelerated clinical trials to develop an effective and safe vaccine to curtail the current ongoing pandemic. The purpose of this review article is to provide an up-to-date status of various promising vaccine platforms.

\section{Genomic Characteristics of SARS-CoV-2}

The genome of SARS-CoV-2 is a positive sense, singlestranded, non-segmented RNA genome of 29,903 nucleotides. The three pathogenic Covs species have several structural and immunological similarities. SARS- CoV-2 genome has $80 \%$ similarity to SARS-CoV and 50\% to MERS-Cov and similar life-cycle shown in Figure 1. The S protein in SARS-CoV-1 is highly conserved in SARS-CoV-2. These similarities help to develop treatment interventions. The genome of SARS- CoV-2 has two open-reading frames (ORFs), ORF1a and ORF1b, which encode the non-structural proteins (nsps) and structural proteins. ORF1a encodes for polypeptide 1a (ppla) that subsequently breakdown into $11 \mathrm{nsps}$. ORF1b initially translated into a polypeptide (pplab) next cleaved into $15 \mathrm{nsps}$ by viral proteases. Another key nsp, viral RNA-dependent RNA polymerase (nsp12), is responsible for replication and transcription of the viral genome. ${ }^{6,7}$

Like all CoV, SARS- CoV-2 encodes four structural proteins which are the $\mathrm{S}, \mathrm{N}, \mathrm{M}$, and $\mathrm{E}$ proteins. The $\mathrm{S}$ protein mostly serves for Covs vaccine development due to its ability to induce neutralizing antibodies and $\mathrm{T}$ cell response. $\mathrm{S}$ protein also mediates viral entry into host cells. S has two domains S1

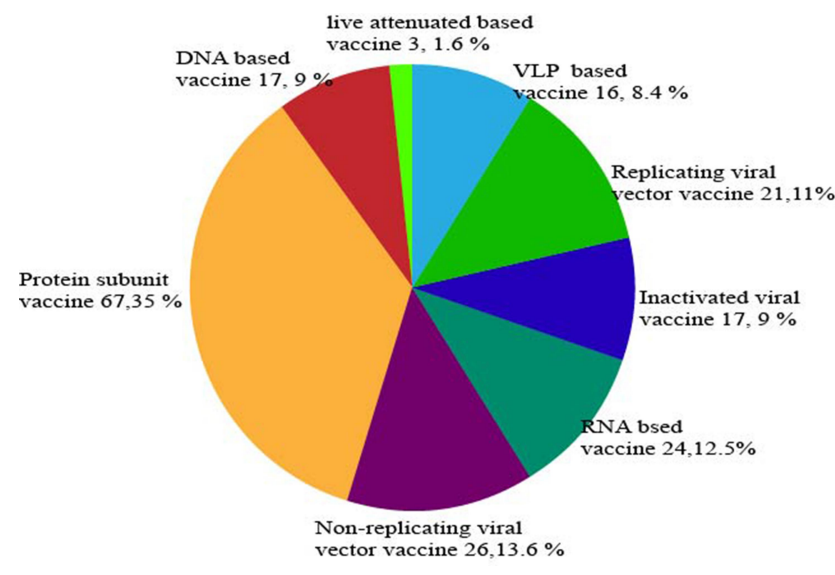

Figure I Distribution of different SARS-CoV-2 vaccine candidate platform. and $\mathrm{S} 2$. The $\mathrm{S} 1$ has the receptor-binding domain (RBD), and the S2 contains the fusion peptide, which is responsible for receptor binding and cell fusion, respectively. ${ }^{8,9}$ Lan et al reported the crystal structure of RBD of SARS-CoV-1 is almost identical to that of SARS-CoV-2. Some mAbs targeting the RBD can neutralize both viruses, making it a promising target for vaccine development with a dual target. For SARSCoV-2, studies with monoclonal antibodies have shown that humans infected with SARS-CoV-2 develop robust neutralizing antibody responses against $\mathrm{S}$ protein and in particular the RBD. ${ }^{10}$ In addition to $\mathrm{S}, \mathrm{N}$-protein-immunized BALB/c mice also induced CD4+ and CD8+ T cells. However, vaccination with vaccines expressing $\mathrm{N}$ resulted in no protection against SARS-CoV challenge as well as enhanced infection, which were characterized by increased pulmonary eosinophil infiltration. The $\mathrm{M}$ and $\mathrm{E}$ proteins have garnered less interest as vaccine targets due to lower immunogenicity, although the SARS-CoV patient sera was shown to be reactive to M peptides. ${ }^{11}$

\section{SARS-CoV-2 Vaccines Under Development}

Vaccination is one of the world's most successful health interventions, saving as many as 3 million lives every year. Vaccination is the cheapest and the most effective way of controlling disease, so efforts are directed towards vaccine designs against COVID-19 to avert the pandemic. Several vaccine candidates have been developed using the S-protein of SARS-CoV-2. ${ }^{12}$ As of December 10, 2020, over 214 candidate vaccines against SARS-CoV-2 from pharmaceutical industries and academic institutions were under development using different vaccine platforms. These vaccine platforms are recombinant viral-vectored vaccines, live attenuated viruses, protein subunit, inactivated vaccines, nucleic acid-based vaccines, and virus-like particles and are presented in Figure 2. Among 214 candidate vaccines, 52 candidate vaccines are in clinical trials. ${ }^{13}$ Currently, Ad5-nCoV (CanSino Biologicals), mRNA-1273 (Moderna), INO-4800 (Inovio, Inc.), BNT162/ mRNA (Fosun Pharma), BBIBP-CorV (Sinopharm), CoronaVac (Sinovac), and ChAdOx1 (University of Oxford) have entered Phase III clinical trials. ${ }^{11,13}$

\section{Protein Subunit Vaccines}

Subunit vaccines may possess antigens with strong immunogenicity that can induce the host immune system. Peptide epitopes isolation is essential for the protein of interest. The epitopes must have the potential to express a strong humoral 


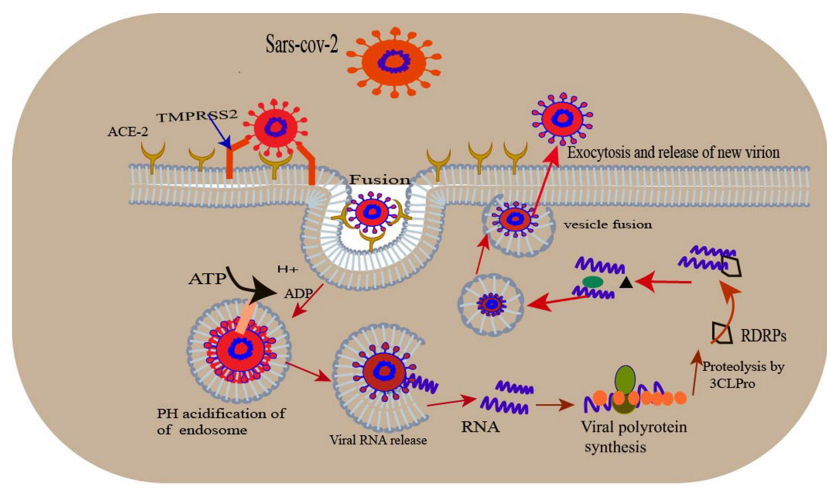

Figure 2 Life cycle of SARS-CoV-2 virus.

and $T$ cell immunity against the pathogen that may have a longlasting effect. ${ }^{14}$ Most subunit vaccines require an adjuvant to induce an enhanced immune response. An adjuvant may increase the half-life of the vaccine or may decrease the immunomodulatory cytokine response. So, an adjuvant overcomes the limitation of the protein subunit vaccines. Research for the development of vaccines for SARS-CoV and MERS-CoV give positive inputs. ${ }^{15}$ Different forms of S-proteins, including the full-length $\mathrm{S}$ protein, $\mathrm{S} 1$, and $\mathrm{RBD}$, displayed a varying degree of neutralizing antibody responses and protection from the virus in several studies. ${ }^{16}$

\section{Vaccines Based on Full-Length S Protein}

Several institutions utilize the subunit vaccines platform to develop the COVID-19 vaccine, especially when using the $\mathrm{S}$ protein as an antigen. The spike (S) protein induces strong immunogenicity, which is the most promising antigen for COVID-19 vaccine development. S protein is the surface protein that mediates virus binding to the host ACE2 receptor for viral fusion and entry. Currently, several vaccine candidates used the $\mathrm{S}$ protein to induce an enhanced immune response. ${ }^{17}$ The $\mathrm{S}$ protein-based vaccines developed against SARS-CoV and MERS-CoV viruses were effective to a large extent. Since SARS$\mathrm{CoV}-2$ shares a considerable genome sequence and sequence identity of different crucial enzymes with SARS$\mathrm{CoV}$, the vaccine approaches already built for SARS may facilitate the development of a COVID-19 vaccine. ${ }^{18}$

NVX-CoV2373 is under development by Novavax from recombinant full-length $\mathrm{S}$ protein with saponinbased Matrix-M1 adjuvant that is Novavax's proprietary nanoparticle technology. Matrix-M induces leukocyte migration into the draining lymph nodes (LN) that increase $\mathrm{T}$ cell, B cell, NK, and dendritic cells in draining to LNs. NVX-CoV2373 produces high levels of S protein-specific antibodies that can block ACE-2 human RBD and wildtype SARS-CoV-2 neutralizing antibodies after one dose. NVX-CoV2373 is in Phase 3 clinical trials in the United Kingdom in combination with the seasonal influenza vaccine. Novavax is currently conducting phase 3 clinical trials in the United Kingdom. ${ }^{19,20}$

\section{Protein (RBD-Based) Subunit Vaccine}

Subunit vaccines based on recombinant antigenic proteins are essential for expressing long-lasting immune responses. RBD of SARS-CoV-S contains major antigenic epitopes that induce both neutralizing antibodies and T cell responses. ${ }^{21}$ The immunogenicity of RBD vaccines was better than the full-length $S$ protein. SARS-CoV RBD did not cause immune damage in the animal model, while the full-length $\mathrm{S}$ protein could do. It is proposed that there are super-antigens in the region beyond the RBD of the full-length S protein. Therefore, the RBD-based subunit vaccine may be the ideal and safer alternative to develop a vaccine for Covs. ${ }^{22,23}$ Kentucky Bioprocessing Inc is developing an RBD-based vaccine candidate and undertaking phases I and II clinical trials (NCT04473690). Anhui Zhifei Longcom Biopharmaceutical/Institute of Microbiology, the Chinese Academy of Science has a candidate vaccine that is currently in phase III clinical trial. ${ }^{24}$

\section{Vaccines Based on S2 Subunit}

SARS-CoV S2 subunit is responsible for fusion between virus and target cell membrane is proposed to be an effective vaccine candidate against SARS-CoV. Several studies showed that S2 protein induces neutralizing antibodies, although their potency is lower than the RBD-based subunit vaccine. However, Zhang et al reported the immune responses against $\mathrm{S} 2$ fragment in $\mathrm{BALB} / \mathrm{c}$ mice induced a specific cellular immune response. ${ }^{25}$ Besides, Zhao et al purified S2-specific IgG from mice immunized with S2 proteins and found that anti-S2 IgGs could abolish the binding between S protein and its cellular receptor(s). Thus, the $\mathrm{S} 2$ subunit has a promising potential to be a target to develop a vaccine against divergent virus strains. Currently, several vaccines are under preclinical studies using this platform. ${ }^{26}$

\section{Inactivated Vaccines}

Inactivated vaccine platforms have been widely used over the past 70 years. Inactivated vaccines are produced by inactivating the viruses with chemicals, UV light, and heat. Inactivation of the organism makes a safe vaccine, especially for immunocompromised persons. However, these vaccines induce a weaker immune response than 
live vaccines and need several booster doses. These inactivated vaccines take a longer time to manufacture as the virus needs to be cultured in the lab and then inactivated. ${ }^{27,28}$ Nowadays, seven inactivated COVID-19 vaccine candidates are in clinical trials, and 12 candidates are in preclinical trials. ${ }^{13}$

CoronaVac, formerly known as PiCoVacc, is an inactivated vaccine candidate developed by the People's Republic of China's leading vaccine manufacturers, Sinovac Biotech. CoronaVac vaccine is made by proliferating the viruses in cell culture followed by inactivation by formalin with alum adjuvant. Inactivated SARS-CoV-2 viruses possess the RBD within the $\mathrm{S}$ protein as immune inducer. $^{28}$ CoronaVac was granted an emergency use authorization by Chinese authorities before the initiation of phase III studies. This authorization resulted in $90 \%$ of company employees immunized with the vaccine. The phase II clinical trial results by CoronaVac showed that it induced neutralizing antibodies 14 days after vaccination. The neutralizing seroconversion rate of CoronaVac was over $90 \%$ in 600 healthy volunteers and can trigger a positive immune response. Currently, CoronaVac is in phase III (NCT04456595) clinical trials. ${ }^{29,30}$

New Crown COVID-19 has been developed by Wuhan Institute of Biological Products and Sinopharm as an inactivated whole-virus, alum-adjuvant vaccine. The whole virus cultivated in vitro and infected cells was further inactivated using $\beta$-propiolactone and adsorbed to $0.5 \mathrm{mg}$ alum. The phase 1 clinical trial was carried out using three doses $(10 \mu \mathrm{g}, 5 \mu \mathrm{g}$, and $2.5 \mu \mathrm{g})$ of antigen. The results revealed that the vaccine has better safety profiles and strong neutralizing antibody response in all three doses. There were no severe side effects. Phase II clinical trials were undertaken using a $5 \mu \mathrm{g}$ antigen, and the results displayed New Crown COVID-19 vaccine can effectively generate antibody titer with fewer side effects. A booster dose is essential to produce enough immune response, with (21 days and 28 days) interval between the first doses. The booster dose triggers a better antibody titer as compared to the 14-day intervals. Generally, the New Crown COVID-19 vaccine candidate displayed acceptable safety and a better immunogenic profile, supporting its assessment in the ongoing phase III trials. A phase III (ChiCTR2000034780) clinical trial began in July 2020 and planned to enroll 21,000 participants. Phase III (ChiCTR2000034780) clinical trials are currently taking place in the United Arab Emirates. Sinopharm gave these candidate vaccines to thousands of people under emergency use conditions approved by the Chinese Government. ${ }^{31-33}$

BBIBP-CorV is another inactivated vaccine candidate developed by the Beijing Institute of Biological Products and Sinopharm. In preclinical studies, BBIBP-CorV produced a better immune response in guinea pigs, mice, rats, rabbits, and non-human primates to protect against SARSCoV-2. In the phase I and II trials, BBIBP-CorV was safe and well-tolerated at all three doses $(2 \mu \mathrm{g}, 4 \mu \mathrm{g}$, or $8 \mu \mathrm{g})$ on days 0 and 28. A robust immune response was observed in $100 \%$ of vaccine recipients. The phase III trial of BBIBP-CorV is ongoing in Abu Dhabi, UAE. ${ }^{34}$

Bharat Biotech's Covaxin is India's first inactivated vaccine candidate developed by Bharat Biotech, Indian Council of Medical Research, and National Institute of Virology against COVID-19. The phase I and II clinical trials were conducted in 12 hospitals in different cities across the country. The vaccine produced robust immune responses, thereby preventing the SARS-CoV-2 virus. Bharat Biotech is also undertaking a phase III clinical trial with 26,000 participants from across 22 sites in India. ${ }^{35}$

\section{Live-Attenuated Vaccines}

Live-attenuated vaccines (LAV) controlled several infections disease outbreaks like yellow fever, mumps, measles, rubella, polio, and chickenpox. The highly attenuated modified vaccinia virus Ankara, recombinant adenoassociated virus, or an attenuated parainfluenza virus encodes recombinant forms of the S protein. ${ }^{36}$ Cao et al evaluated the immune protection of a rAAV encoding RBD vaccine in a mouse model by intranasal inoculation, which induced strong mucosal immune responses and provided long-term protection against SARS-CoV infection. ${ }^{37}$ Other groups reported that immunization of monkeys via the respiratory tract with BHPIV3/SARS-S induced the production of SARS-CoV-neutralizing serum antibodies. Weingart et al immunized ferrets with rMVA-S and the immunized ferrets induced neutralizing antibody. After challenging with SARS-CoV, ferrets showed strong inflammatory responses in liver tissue, suggesting that vaccination with rMVA-S enhances hepatitis, which is not observed in the above infection model. It is suggested that we should not only use mice or monkeys as an animal model but also use ferrets as the infection model, because of their sensitivity to SARS-CoV. ${ }^{38}$ LAVs associate with the risk of reversion by either mutation- or recombinationdriven processes, which cause dangerous outbreaks in unvaccinated populations. 
LAV vaccines against SARS-CoV-2 are currently in preclinical development. For example, Indian Immunologicals Limited is currently working together with Griffith University to develop a vaccine using codon deoptimization as a strategy against SARS-CoV-2. The vaccine candidate provides a long-lasting immunity against SARS-CoV-2 following a single vaccination. ${ }^{39}$

DelNS1-SARS-CoV2-RBD is an influenza-based vaccine strain with deletion of the virulent NS1 gene. It is attenuated by the deletion of a virulent element and the immune antagonist. It is more immunogenic than the wildtype influenza virus and can be given as a nasal spray. An attenuated version of the influenza virus was modified to encode the RBD domain of SARS-CoV-2 spike protein on its surface. ${ }^{40}$

\section{Nucleic Acid-Based Vaccines (DNA or mRNA) Platform}

Nucleic acid-based vaccines offer a cost-effective approach to SARS-CoV-2 vaccine development. In the 1990s Wolf et al reported in vivo the encoded protein expression after administration of encoded nucleic acids (RNA or DNA) into mice. ${ }^{41}$ This discovery was the start of the use of nucleic acids encoding antigens as a form of vaccination. While the instability of mRNA limited its use, plasmid DNA emerged as the promising platform even though the first clinical trial was disappointing. Advances in the delivery of vaccines have spurred new clinical trials to develop vaccines against Covid-19. Currently, several companies are developing nucleic acid-base d vaccines. ${ }^{42,43}$

\section{mRNA Vaccine}

The mRNA-based vaccine is an emerging, non-infectious, and non-integrating platform with less risk of insertional mutagenesis. It is the most promising alternative due to its cost and safety profile in animal studies. The immunogenicity of the mRNA can be minimized, and alterations can increase the stability of these vaccines. Furthermore, the anti-vector immunity can be removed as the mRNA is the minimally immunogenic genetic vector, allowing repeated use of the vaccine. This platform has empowered the rapid vaccine development program due to its flexibility and ability to mimic the antigen structure and expression as seen in the course of natural infection. ${ }^{44,45}$ Currently, six mRNA-based vaccine candidates are in the clinical trials, and 19 vaccine candidates are in the preclinical trial for COVID-19. ${ }^{13}$

mRNA-1273 is an mRNA vaccine with a synthetic viral mRNA, which encodes the full-length spike protein (S) of SARS-CoV-2 and mimics the natural infection. The host body recognizes the synthetic viral mRNA and translates the viral protein shown in Figure 3. mRNA is not stable, but through chemical modifications, mRNA is stabilized and packaged into an injectable form using a liquid nanoparticle. ${ }^{44-46}$ The mRNA-1273 is a lipid nanoparticle (LNP)-encapsulated mRNA-based vaccine that encodes a stable form of the spike protein of SARS-CoV-2. The encapsulated mRNA-1273 then travels to the immune cells (lymph nodes) and instructs them to make copies of the spike protein on their surface as if SARS-CoV-2 infects them. Other immune cells learn about the spike protein and prepare themselves for the future response to SARSCoV-2. ${ }^{47}$ mRNA-1273 evoked neutralizing antibody titer levels observed in convalescent sera within their initial 45 participants. It is also generally safe and provided complete protection in a mouse challenge model. ${ }^{48}$ Moderna has collaborated with Catalent since June 25, 2020 to perform large-scale, commercial fill-finish manufacturing of Moderna's mRNA-1273 COVID-19 vaccine at Catalent's biologics facility in Indiana, US. Besides, it is relatively safe as it is neither an inactivated pathogen nor the subunits of the live pathogen. mRNA used in vaccination is safe to use since it cannot become part of a person's chromosomes. ${ }^{47,48}$ Most adverse events were mild or moderate, with Grade 3 (severe) in $2 \%$, typically pain at the injection site and fatigue, achiness, joint pain, headache, and redness at the injection site after the second dose. Moderna reported that the mRNA-1273 vaccine candidate passed its most important test, the 30,000+ participant phase III clinical trial (NCT04470427) by showing 94.5\% effectiveness in preventing COVID-19. The FDA

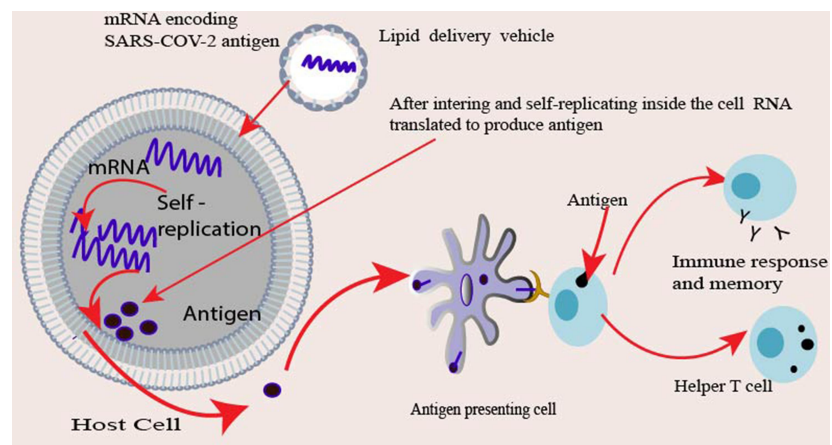

Figure 3 RNA vaccine platform. 
advisory panel voted to recommend the approval of the mRNA-1273 vaccine for emergency use. ${ }^{49}$

BNT162b1/BNT162b2 vaccines are codon-optimized mRNA vaccines that encode SARS-CoV-2 RBD and fulllength spike respectively. The mRNA is encapsulated in $80 \mathrm{~nm}$ ionizable cationic lipid nanoparticles that increase its delivery. The phase II and III clinical trials showed the two vaccine candidates elicited similar dose-dependent SARS-CoV-2-neutralizing geometric mean titers (GMTs), comparable to the GMT of a panel of SARS - CoV-2 convalescent sera. BNT162b2 showed less systemic reactogenicity, particularly in older adults. ${ }^{50}$

The ongoing phase 3 clinical trial of BNT162b2, has enrolled more than 44,000 participants, from 150 sites in the US, Germany, Turkey, South Africa, Brazil, and Argentina. Most participants have received their second dose. The phase III trial BNT162b2 was 95\% effective 28 days after the first dose. FDA granted Pfizer and BioNTech an emergency use authorization (EUA) of the BNT162b2 COVID-19 vaccine. ${ }^{51}$

THe LUNAR-COV19 (ARCT-021) vaccine contains a lipid-mediated delivery system called Lipid-enabled and Unlocked Nucleomonomer Agent-modified RNA (LUNAR). After administration, the vaccine enters the host's cells, where the mRNA translates into proteins. These proteins are from the SARS-CoV-2 virus, and thus the host can induce an immune response against them and fight off an infection from the native virus. It is developed by Arcturus Therapeutics' on an RNA-based vaccine platform and enters phase I and II clinical trials in Singapore. $^{52}$

\section{DNA Vaccines}

The most revolutionary approach to vaccination is the introduction of the DNA vaccine, which encodes antigen, and induces the adaptive immune response. The transfected cells express the transgene that provides a steady supply of the transgene-specific proteins, which is similar to the live virus. ${ }^{53}$ DNA vaccines targeting $\mathrm{S}, \mathrm{M}$, and $\mathrm{N}$ proteins induce humoral as well as cellular immune response. Although the DNA vaccine platform is temperature stable and rapidly manufactured, their efficacy and immunogenicity in persons are not yet proven. DNA vaccine administration, vector mutations, and genome integration into the host gene are remaining issues. DNA vaccine platform was started in 1993 with promising results against influenza viruses, but the same results cannot be translated to humans yet. ${ }^{54}$
INO-4800 is a DNA vaccine candidate developed by Inovio Pharmaceuticals. It was designed to optimize the $\mathrm{S}$ protein sequence of the SARS-CoV-2 virus. The presence of humoral and $\mathrm{T}$ cell response in the preclinical trials suggest that $I N O-4800$ can produce an effective immune response. The vaccine has entered phase I and II (NCT04336410) clinical trials. The clinical trial will evaluate the immunological profile, safety, and tolerability of the vaccine candidate. ${ }^{55}$

\section{Replicating and Non-Replicating Viral Vectors Vaccines}

Viral vectors vaccine is a promising prophylactic solution against a pathogen. These vaccines are specific in delivering the genes to the target cells, highly efficient in the gene transduction, and induce the immune response. Viral vectors vaccine provides prolonged time and enhanced antigenic protein expression and, therefore, has better prophylactic use as these vaccines trigger the cytotoxic $\mathrm{T}$ cells, which leads to the removal of the virus-infected cells. Viral vectors are grouped into replicating (measles virus and vesicular stomatitis virus) or non-replicating vectors (Adenoviruses (Ad) and poxviruses). Several vaccine developments rely on non-replicating Ads vector. These vectors are relatively safe, physically, and genetically stable, and do not integrate into the host genome. Ads vector can infect DCs and dividing or non-dividing cells. However, Ad vectors need high doses to induce the host immune response. ${ }^{56,57}$

AZD1222 (ChAdOx1 nCoV-19): is a SARS-CoV-2 vaccine candidate that uses a non-replicating chimpanzee adenovirus as a vector (ChAdOx1) and is modified to induce the $\mathrm{S}$ protein from SARS-CoV-2. AZD1222 is under development by the University of Oxford and AstraZeneca. This adenovirus is genetically modified so that it does not replicate in humans. ${ }^{53}$ After vaccination, cells induce spike protein and the critical immune system to produce neutralizing antibodies that bind to spike glycoprotein and attack SARS-Cov-2 virus. Besides, the vaccine induces T-cells, which can attack the host cells if they get infected by the SARS-CoV-2 virus. Phase I and II studies show that the candidate AZD1222 vaccine given at a dose of $5 \times 10^{10}$ viral particles was safe and tolerated, however higher reactogenicity was displayed than the control vaccine. The reactogenicity decreased with 1 g paracetamol for the first 24 hours after vaccination. Generally, the two doses were enough to induce a potent 
immune response in all the participants and had no severe adverse reactions. Phase III trials are currently ongoing in several countries. ${ }^{56-59}$

On June 29, 2020, the People's Republic of China's military received permission to use the Ad5-nCoV vaccine (Adenovirus vaccine) candidate developed by the People's Republic of China's CanSino Biologics Inc. as it was proved safe and efficient in the clinical trials (NCT04341389). Ad5-nCoV uses replication-defective human Ad5 as a vector and is modified to express the $\mathrm{S}$ protein. This vector was used for the Ebola vaccine (Ad5-EBO) development. Ad5-nCoV vaccine candidate developed by the People's Republic of China's CanSino Biologics Inc. as it was proved safe and efficient in the clinical trials (NCT04341389). The phase I clinical trials showed four-fold in RBD and S protein-specific neutralizing antibodies and specific $\mathrm{T}$ cell responses. However, the pre-existing anti-Ad5 immunity may antagonize both the antibody and the $\mathrm{T}$ cell responses before an immune response to the $\mathrm{S}$ protein develops. ${ }^{60,61}$

Coroflu (M2SR) is a unique influenza virus. It lacks a gene called M2 that limits the influenza virus to undergo only a single replication in cells. M2SR is modified by the insertion of the spike protein gene sequence of the SARS$\mathrm{CoV}-2$. It can enter into the host cell, thereby inducing an immune response against the SARS-CoV-2. It is administered intra-nasally, mimicking the natural route of viral infection. The immune response is higher as compared to the intramuscular injections. It is in the preclinical phase. ${ }^{62}$

LV-SMENP-DC vaccine: Dendritic cells are antigenpresenting cells which induce immune response via antigen presentation. Dendritic cells exist in the periphery and are active at engulfing and presenting exogenous antigens. LV-SMENP-DC vaccine is developed by modifying dendritic cells and $\mathrm{T}$ cell activation by the lentiviral vector to encode COVID-19 antigen. The subcutaneous inoculations of the vaccine present the cytotoxic $\mathrm{T}$ cells and generate the immune response. LV-SMENP is currently undergoing a phase I and II multicenter trial (NCT04276896) in healthy volunteers. ${ }^{63,64}$

On August 11, 2020, Russia approved the "Sputnik V" anti-SARS-CoV-2 vaccine developed by Moscow's Gamaleya Institute. It is not clear if the completion of all phases of clinical trials were carried out. Phase III clinical trial for its effectiveness would be done after regulatory approval. Sputnik V uses a non-replicating viral vector "adenovirus" containing gene-specific spike protein like other vaccine candidates. The difference with other candidate vaccines is that Sputnik V uses two adenovirus vectors: Ad26 and Ad5, instead of a single serotype. In the first dose, Ad26 is given and, the second dose of Ad5 is administered after 21 days. This strategy has an advantage as, after the first dose, antibodies are produced against the Ad26 serotype. The second dose is of Ad5 serotype; therefore, the body is stimulated to produce an enhanced immune response. Phase I and II trial results of Sputnik $\mathrm{V}$ have shown antibody production in all the trial participants, though the sample size was small, and further studies will be carried out in the elderly. ${ }^{65-68}$ After the announcement of Sputnik-V, the Russian government received preliminary orders for greater than 1 billion doses of Sputnik V vaccine from 20 countries. Now, Russia is manufacturing more than 500 million doses of Sputnik V vaccine. ${ }^{68}$

\section{Virus-Like Particle Vaccines}

VLPs mimic the conformation of native viruses. VLPs contain almost all viral proteins, but lack a viral genome that causes disease transmission and non-structural proteins. Due to a lack of genetic material, VLPs cannot replicate in the host but can express both the cellular and humoral immune responses. VLP can be produced using a combination of structural proteins from various types of viruses and plants. Currently, several VLP-based vaccines are available such as HBV and HPV vaccines for prophylactic use. $^{69}$

Plants are an ideal platform for oral vaccine production. The genome for viral proteins is delivered to the plant using Agrobacterium bacteria. After infection, the gene of interest integrates into the plant genome and produce virus-like particles. Plant-based vaccines produced using Agrobacterium and Nicotiana benthamiana against influenza A viruses (A/ H1N1, A/H3N2), and avian influenza H5 (AIV) were effective, safe, and well-tolerated. This vaccine platform was displayed as a promising source of vaccine for Lyme disease, Newcastle disease, bovine viral diarrhea virus, and recombinant colicin M. Several studies proposed different recombinant VLP vaccines against SARS-CoV-2 viruses. ${ }^{69,70}$

Currently, several VLP vaccines under development are RBD SARS-CoV-2 HBsAg VLP vaccine in phase I and II (ACTRN12620000817943) developed by SpyBiotech and Serum Institute of India, and CoVLP is in phase II/III (NCT04636697) developed by Medicago biopharmaceutical in Canada. The Medicago Inc. vaccine was developed by using its proprietary plant-based technology using tobacco plants to produce virus-like particles. 
Table I The Most Promising Vaccine Candidates That Have Recently Moved into Human Phase

\begin{tabular}{|c|c|c|c|}
\hline $\begin{array}{l}\text { Vaccine } \\
\text { Platform }\end{array}$ & Candidate & Clinical Trial Stage & Developer \\
\hline \multirow{4}{*}{$\begin{array}{l}\text { Viral } \\
\text { vector-based } \\
\text { vaccines }\end{array}$} & $\mathrm{Ad5}-\mathrm{nCoV}$ & $\begin{array}{l}\text { Phase } 2 \text { ChiCTR200003I } 78 \text { I } \\
\text { Phase I ChiCTR2000030906 }\end{array}$ & $\begin{array}{l}\text { CanSino Biological Inc./Beijing } \\
\text { Institute of Bio-technology }\end{array}$ \\
\hline & AZDI222 (ChAdOxI nCoV-19) & $\begin{array}{l}\text { Phase 3: ISRCTN8995I } 424 \\
\text { Phase2b/3: NCT04324606 }\end{array}$ & University of Oxford/AstraZeneca \\
\hline & $\begin{array}{l}\text { Lenti viral based Minigene dendritic cell (DC) and } \\
T \text { cell vaccine (LV-SMENP-DC) }\end{array}$ & Phase I: NCT04276896 & $\begin{array}{l}\text { Shenzhen Geno-Immune Medical } \\
\text { Institute }\end{array}$ \\
\hline & Adeno-based Gam-COVID-Vac & $\begin{array}{l}\text { Phase I: NCT0443647I } \\
\text { NCT04437875 }\end{array}$ & Gamaleya Research Institute \\
\hline \multirow[t]{4}{*}{$\begin{array}{l}\text { Inactivated } \\
\text { virus vaccines }\end{array}$} & Inactivated viral vaccine & $\begin{array}{l}\text { Phase I/2: } \\
\text { ChiCTR2000031809 }\end{array}$ & $\begin{array}{l}\text { Wuhan Institute of Biological } \\
\text { Products/Sinopharm }\end{array}$ \\
\hline & CoronaVac & Phase 3 (NCT04470427) & Sinovac \\
\hline & New Crown & Phase 3 (ChiCTR2000034780) & $\begin{array}{l}\text { Wuhan Institute of Biological } \\
\text { Products, Sinopharm }\end{array}$ \\
\hline & BBIBP-CorV & Phase 3 (ChiCTR2000034780) & $\begin{array}{l}\text { Beijing Institute of Biological } \\
\text { Products, Sinopharm }\end{array}$ \\
\hline $\begin{array}{l}\text { DNA } \\
\text { vaccines }\end{array}$ & INO-4800 & Phase I NCT043364I0 & Inovio Pharmaceuticals \\
\hline \multirow[t]{4}{*}{ RNA vaccines } & mRNA-I 273 & Phase 3 (NCT04470427) & Moderna/NIAID \\
\hline & BNTI62/mRNA & Phase 3 (NCT04368728) & BioNTech| FosunPharma| Pfizer \\
\hline & Cure Vac mRNA/RABV & Phase I(NCT04449276) & CureVac \\
\hline & LNP-nCoVsaRNA/RNA/EBOV & Phase I: ISRCTNI 7072692 & Imperial College London \\
\hline $\begin{array}{l}\text { Protein } \\
\text { subunit } \\
\text { vaccine }\end{array}$ & NVX-(CoV2373)/RSV & Phase I/2: NCT04368988 & Novavax | Emergent BioSolutions \\
\hline
\end{tabular}

The RBD-HBsAg-VLPs-Covid vaccine based on the RBD domain of SARS-CoV-2 conjugated to the hepatitis B surface antigen (HBsAg) virus-like particles. ${ }^{71,72}$

\section{Conclusion}

A vaccine is necessary to end the world's SARS-CoV-2 pandemic. A successful vaccine is safe, effective, durable, and deployable to large populations. RNA viruses may mutate and this may make previously effective vaccines useless. Thus, development of a safe and effective vaccine against SARS-CoV strains is crucial for protecting future SARS-CoV outbreaks. A broad spectrum SARS vaccine that is effective against all the Covses using ACE2 as their receptor can be applied immediately when we face the recurrent threat of Covs. Particularly, taking SARS-CoV-2 as an example, the virus might disappear before the vaccine is developed. Therefore, development of safe and broadspectrum vaccines against SARS-CoV-2 and other SARSCoV-related viruses is of great significance for preventing humans from $\mathrm{CoV}$ infection. Most vaccine strategies aim to generate S-specific neutralizing antibodies. Vaccines based on RBD of $\mathrm{S}$ protein against SARS-CoV may efficiently cross neutralize SARS-CoV-2. Besides antigens, adjuvant can increase the immune response and reduce the amount of antigen needed for each dose of the vaccine.

As of December 10, 2020, over 214 candidate vaccines are under development and 52 candidate vaccines are in clinical trials as shown in Table 1 and seven of them have entered phase 3 trials. Several of the candidates have been judged safe enough for use in humans and have shown 
substantial protection against SARS-CoV-2. Over the next several months, additional studies will be undertaken on immune responses providing protection against COVID19. Throughout history, several vaccine candidates have disappointed when their protective effect was evaluated in large clinical trials. It is well documented that vaccines which show promise in phase I and II trials, are found to be ineffective in phase III trials.

\section{Abbreviations}

LAV, live-attenuated vaccines; MERS-CoV, Middle East respiratory syndrome coronavirus; nsps, non-structural proteins; Nabs, neutralizing monoclonal antibodies; ORFs, open reading frames; RdRp, RNA-dependent RNA polymerase; SARS-CoV, severe acute respiratory syndrome coronavirus; SARS-CoV-2, severe acute respiratory syndrome coronavirus-2.

\section{Data Sharing Statement}

All data are provided in the article or found from published papers as cited.

\section{Acknowledgment}

I would like to acknowledge Mrs Fasika Abu for editing the manuscript for English Style.

\section{Disclosure}

The author declares no conflicts of interest for this work.

\section{References}

1. Sharma A, Tiwari S, Deb MK, Marty JL. Severe Acute Respiratory Syndrome Coronavirus-2 (SARS-CoV-2): a global pandemic and treatments strategies. Int $J$ Antimicrob Agents. 2020;10:106054. doi:10.1016/j.ijantimicag.2020.106054

2. Wang N, Shang J, Jiang S, Du L. Subunit vaccines against emerging pathogenic human coronaviruses. Front Microbiol. 2020;28(11):298. doi: $10.3389 /$ fmicb. 2020.00298

3. World Health Organization. WHO Coronavirus Disease (COVID-19) Dashboard. Available from: https://covid19.who.int/. Accessed 15 December, 2020.

4. Cao Q, Chen YC, Chen CL, Chiu CH. SARS-CoV-2 infection in children: transmission dynamics and clinical characteristics. $J$ Formosan Med Assoc. 2020;119(3):670. doi:10.1016/j. jfma.2020.02.009

5. Samrat SK, Tharappel AM, Li Z, Li H. Prospect of SARS-CoV-2 spike protein: potential role in vaccine and therapeutic development. Virus Res. 2020;23:198141. doi:10.1016/j.virusres.2020.198141

6. Helmy YA, Fawzy M, Elaswad A, Sobieh A, Kenney SP, Shehata AA. The COVID-19 pandemic: a comprehensive review of taxonomy, genetics, epidemiology, diagnosis, treatment, and control. J Clin Med. 2020;9(4):1225. doi:10.3390/jcm9041225
7. Andersen KG, Rambaut A, Lipkin WI, Holmes EC, Garry RF. The proximal origin of SARS-CoV-2. Nat Med. 2020;26(4):450-452. doi:10.1038/s41591-020-0820-9

8. Zhang YZ, Holmes EC. A genomic perspective on the origin and emergence of SARS-CoV-2. Cell. 2020;181(2):223-227. doi:10.1016/j.cell.2020.03.035

9. Belete TM. An up-to-date overview of therapeutic agents for the treatment of COVID-19 disease. Clin Pharmacol. 2020;12:203-212. doi:10.2147/CPAA.S284809

10. Wajnberg A, Amanat F, Firpo A, et al. SARS-CoV-2 infection induces robust, neutralizing antibody responses that are stable for at least three months. MedRxiv. 2020.

11. Dagotto G, Yu J, Barouch DH. Approaches and challenges in SARS-CoV-2 vaccine development. Cell Host Microbe. 2020;28 (3):364-370. doi:10.1016/j.chom.2020.08.002

12. Hadjipanayis A, van Esso D, Del Torso S, et al. Vaccine confidence among parents: large scale study in eighteen European countries. Vaccine. 2020;38(6):1505-1512. doi:10.1016/j.vaccine.2019.11.068

13. World Health Organization. DRAFT Landscape of COVID-19 Candidate Vaccines. Geneva: WHO; 2020.

14. Baviskar T, Raut D, Bhatt LK. Deciphering vaccines for COVID-19: where do we stand today? Immunopharmacol Immunotoxicol. 2020;14:1-48.

15. Baviskar T, Raut D, Bhatt LK. Deciphering vaccines for COVID-19: where do we stand today? Immunopharmacol Immunotoxicol. 2020;23:1-4

16. Belete TM. A review on promising vaccine development progress for COVID-19 disease. Vacunas. 2020;21(2):121-128. doi:10.1016/j. vacun.2020.05.002

17. Wu D, Koganti R, Lambe UP, Yadavalli T, Nandi SS, Shukla D. Vaccines and therapies in development for SARS-CoV-2 infections. J Clin Med. 2020;9(6):1885. doi:10.3390/jcm9061885

18. Morse JS, Lalonde T, Xu S, Liu W. Learning from the past: possible urgent prevention and treatment options for severe acute respiratory infections caused by 2019-nCoV. Chem Biochem. 2020;21 (5):730-738. doi:10.1002/cbic.202000047

19. Keech C, Albert G, Cho I, et al. Phase 1-2 trial of a SARS-CoV-2 recombinant spike protein nanoparticle vaccine. $N$ Engl $\mathrm{J}$ Med. 2020;383(24):2320-2332. doi:10.1056/NEJMoa2026920

20. Guebre-Xabier M, Patel N, Tian JH, et al. NVX-CoV2373 vaccine protects cynomolgus macaque upper and lower airways against SARS-CoV-2 challenge. bioRxiv. 2020.

21. Skwarczynski M, Toth I, editors. Micro-And Nanotechnology in Vaccine Development. William Andrew; 2016.

22. Wang J, Peng Y, Xu H. The COVID-19 vaccine race: challenges and opportunities in vaccine formulation.

23. Dai L, Zheng T, Xu K, et al. A universal design of betacoronavirus vaccines against COVID-19, MERS, and SARS. Cell. 2020;182 (3):722-733. doi:10.1016/j.cell.2020.06.035

24. Sumirtanurdin R, MI B. Coronavirus disease 2019 vaccine development: an overview. Viral Immunol. 2020:23.

25. Zhang H, Wang G, Li J, et al. Identification of an antigenic determinant on the S2 domain of the severe acute respiratory syndrome coronavirus spike glycoprotein capable of inducing neutralizing antibodies. $J$ Virol. 2004;78(13):6938-6945. doi:10.1128/ JVI.78.13.6938-6945.2004

26. Zhao P, Ke JS, Qin ZL, et al. DNA vaccine of SARS-Cov S gene induces antibody response in mice. Acta Biochim Biophys Sin (Shanghai). 2004;36(1):37-41. doi:10.1093/abbs/36.1.37

27. Jeyanathan M, Afkhami S, Smaill F, Miller MS, Lichty BD, Xing Z. Immunological considerations for COVID-19 vaccine strategies. Nat Rev Immunol. 2020;4:1-8.

28. Xia S, Duan K, Zhang Y, et al. Effect of an inactivated vaccine against SARS-CoV-2 on safety and immunogenicity outcomes: interim analysis of 2 randomized clinical trials. JAMA. 2020;324 (10):951-960. 
29. Lim HX, Lim J, Jazayeri SD, Poppema S, Poh CL. Development of multi-epitope peptide-based vaccines against SARS-CoV-2. Biomed J. 2020. doi:10.1016/j.bj.2020.09.005

30. Zhang YJ, Zeng G, Pan HX, et al. Immunogenicity and safety of a SARS-CoV-2 inactivated vaccine in healthy adults aged $18-59$ years: report of the randomized, double-blind, and placebo-controlled phase 2 clinical trial. medRxiv. 2020.

31. Chakraborty R, Parvez S. COVID-19: an overview of the current pharmacological interventions, vaccines, and clinical trials. Biochem Pharmacol. 2020;30:114184. doi:10.1016/j.bcp.2020.114184

32. Xia S, Duan K, Zhang Y, et al. Effect of an inactivated vaccine against SARS-CoV-2 on safety and immunogenicity outcomes: interim analysis of 2 randomized clinical trials. JAMA. 2020;324 (10):951-960.

33. Rawat K, Kumari P, Saha L. COVID-19 vaccine: a recent update in pipeline vaccines, their design and development strategies. Eur J Pharmacol. 2020;25:173751.

34. Xia S, Zhang Y, Wang Y, et al. Safety and immunogenicity of an inactivated SARS-CoV-2 vaccine, BBIBP-CorV: a randomised, double-blind, placebo-controlled, phase 1/2 trial. Lancet Infect Dis. 2020;21(1):15. doi:10.1016/S1473-3099(20)30831-8

35. Dutta AK. Vaccine against Covid-19 disease-present status of development. Indian J Pediatrics. 2020;3:1-7.

36. Dai X, Xiong Y, Li N, Jian C. Vaccine Types Vaccines-The History and Future. 2019

37. Cao Z, Liu L, Du L, et al. Potent and persistent antibody responses against the receptor-binding domain of SARS-CoV spike protein in recovered patients. Virol J. 2010;7(1):1-6. doi:10.1186/1743-422X7-299

38. Weingartl $\mathrm{H}$, Czub M, Czub S, et al. Immunization with modified vaccinia virus Ankara-based recombinant vaccine against severe acute respiratory syndrome is associated with enhanced hepatitis in ferrets. $J$ Virol. 2004;78(22):12672-12676. doi:10.1128/ JVI.78.22.12672-12676.2004

39. $\mathrm{Ng} \mathrm{WH}$, Liu $\mathrm{X}$, Mahalingam S. Development of vaccines for SARS-CoV-2. F1000Research. 2020;9.

40. Kaur SP, Gupta V. COVID-19 Vaccine: a comprehensive status report. Virus Res. 2020;13:198114. doi:10.1016/j.virusres.2020.198114

41. Smith TR, Schultheis K, Broderick KE. Nucleic acid-based vaccines targeting respiratory syncytial virus: delivering the goods. Hum Vaccin Immunother. 2017;13(11):2626-2629. doi:10.1080/ 21645515.2017.1363134

42. Piyush R, Rajarshi K, Chatterjee A, Khan R, Ray S. Nucleic acid-based therapy for coronavirus disease 2019. Heliyon. 2020;19: e05007. doi:10.1016/j.heliyon.2020.e05007

43. Chung YH, Beiss V, Fiering SN, Steinmetz NF. COVID-19 vaccine frontrunners and their nanotechnology design. ACS Nano. 2020;14 (10):12522-12537. doi:10.1021/acsnano.0c07197

44. Jackson LA, Anderson EJ, Rouphael NG, et al. An mRNA vaccine against SARS-CoV-2 - preliminary report. $N$ Engl J Med. 2020;383 (20):1920-1931. doi:10.1056/NEJMoa2022483

45. Wang F, Kream RM, Stefano GB. An evidence based perspective on mRNA-SARS-CoV-2 vaccine development. Med Sci Monitor. 2020;26:e924700-1. doi:10.12659/MSM.924700

46. Corbett KS, Flynn B, Foulds KE, et al. Evaluation of the mRNA-1273 vaccine against SARS-CoV-2 in nonhuman primates. $N$ Engl $J$ Med. 2020;383(16):1544-1555. doi:10.1056/NEJMoa2024671

47. Saini P. COVID-19 pandemic: potential phase III vaccines in development. T Appl Biol Chem J. 2020;1(1):21-33.

48. Weide B, Carralot JP, Reese A, et al. Results of the first phase I/II clinical vaccination trial with direct injection of mRNA. J Immunother. 2008;31(2):180-188. doi:10.1097/CJI.0b013e31815ce501

49. Mahase E. Covid-19: moderna vaccine is nearly $95 \%$ effective, trial involving high risk and elderly people shows. $B M J$. 2020;17:371.
50. Walsh EE, Frenck R, Falsey AR, et al. RNA-based COVID-19 vaccine BNT162b2 selected for a pivotal efficacy study. Medrxiv. 2020. doi:10.1101/2020.08.17.20176651

51. Mahase E. Covid-19: UK Approves Pfizer and BioNTech Vaccine with Rollout Due to Start Next Week.

52. Banday AH, Shameem SA, Ajaz SJ. Potential repurposed therapeutics and new vaccines against COVID-19 and their clinical status. SLAS DISCOVERY. 2020;25(10):1097-1107.

53. Gary EN, Weiner DB. DNA vaccines: prime time is now. Curr Opin Immunol. 2020;65:21-27. doi:10.1016/j.coi.2020.01.006

54. Chen WH, Strych U, Hotez PJ, Bottazzi ME. The SARSCoV- 2 vaccine pipeline: an overview. Current Tropical Med Rep. 2020;7 (2):61-64. doi:10.1007/s40475-020-00201-6

55. ClinicalTrials.gov. Identifier: NCT04336410. 2020. Available from: https://clinicaltrials.gov/ct2/show/NCT04336410. Accessed May 2, 2020

56. Shirley JL, de Jong YP, Terhorst C, Herzog RW. Immune responses to viral gene therapy vectors. Mol Ther. 2020;28(3):709-722. doi:10.1016/j.ymthe.2020.01.001

57. Graham SP, McLean RK, Spencer AJ, et al. Evaluation of the immunogenicity of prime-boost vaccination with the replication-deficient viral vectored COVID-19 vaccine candidate ChAdOx1 nCoV-19. NPJ Vaccines. 2020;5(1):1-6. doi:10.1038/ s41541-020-00221-3

58. Folegatti PM, Ewer KJ, Aley PK, et al. Safety and immunogenicity of the ChAdOx1 nCoV-19 vaccine against SARS-CoV-2: a preliminary report of a phase $1 / 2$, single-blind, randomised controlled trial. The Lancet. 2020;396(10249):467-478. doi:10.1016/ S0140-6736(20)31604-4

59. Xia S, Duan K, Zhang Y, et al. Effect of an inactivated vaccine against SARS-CoV-2 on safety and immunogenicity outcomes: interim analysis of 2 randomized clinical trials. JAMA. 2020;324 (10):951-960.

60. Zhu FC, Guan XH, Li YH, et al. Immunogenicity and safety of a recombinant adenovirus type-5-vectored COVID-19 vaccine in healthy adults aged 18 years or older: a randomised, double-blind, placebo-controlled, phase 2 trial. The Lancet. 2020;396 (10249):479-488. doi:10.1016/S0140-6736(20)31605-6

61. Alturki SO, Alturki SO, Connors J, et al. The 2020 Pandemic: current SARS-CoV-2 vaccine development. Front Immunol. 2020;11.

62. Covid-vaccine-tracker, COVID-19 Vaccine \& Therapeutics Tracker, 2020. Available from: https://biorender.com/covid-vaccine-tracker /details/v-0CV3/bacille-calmette-guerin-bcg. Accessed May 2, 2020

63. Siddique R, Bai Q, Shereen MA, et al. Evidence and speculations: vaccines and therapeutic options for COVID-19 pandemic. Hum Vaccin Immunother. 2020;5:1-9. doi:10.1080/ 21645515.2020.1824497

64. Bhagavathula AS, Aldhaleei WA, Rovetta A, Rahmani J. Vaccines and drug therapeutics to lock down novel Coronavirus Disease 2019 (COVID-19): a systematic review of clinical trials. Cureus. 2020;12:5.

65. Petersen E, Wejse C, Zumla A. Advancing COVID-19 vaccinesavoiding different regulatory standards for different vaccines and need for open and transparent data sharing. Int $J$ Infect Dis. 2020;98:501-502. doi:10.1016/j.ijid.2020.08.043

66. Balakrishnan VS. The arrival of Sputnik V. Lancet Infect Dis. 2020;20(10):1128. doi:10.1016/S1473-3099(20)30709-X

67. Burki TK. The Russian vaccine for COVID-19. Lancet Respir Med. 2020;5.

68. Advancing COVID-19 vaccines - avoiding different regulatory standards for different vaccines and need for open and transparent data sharing.

69. van Riel D, de Wit E. Next-generation vaccine platforms for COVID-19. Nat Mater. 2020;19(8):810-812. doi:10.1038/s41563020-0746-0 
70. Karpiński TM, Ożarowski M, Seremak-Mrozikiewicz A, Wolski H, Wlodkowic D. The 2020 race towards SARS-CoV-2 specific vaccines.

71. Bakhiet M, Taurin S. SARS-CoV-2: targeted managements and vaccine development. Cytokine Growth Factor Rev. 2020. doi:10.1016/j.cytogfr.2020.11.001
72. Medicago announces production of a viable vaccine candidate for COVID-19. BioSpace. Available from: https://www.biospace.com/ article/releases/medicago-announces-production-of-a-viablevaccine-candidate-for-covid-19/. Accessed May 24, 2020

\section{Publish your work in this journal}

Infection and Drug Resistance is an international, peer-reviewed openaccess journal that focuses on the optimal treatment of infection (bacterial, fungal and viral) and the development and institution of preventive strategies to minimize the development and spread of resistance. The journal is specifically concerned with the epidemiology of

Submit your manuscript here: https://www.dovepress.com/infection-and-drug-resistance-journal antibiotic resistance and the mechanisms of resistance development and diffusion in both hospitals and the community. The manuscript management system is completely online and includes a very quick and fair peerreview system, which is all easy to use. Visit http://www.dovepress.com/ testimonials.php to read real quotes from published authors. 